\title{
ASSESSMENT OF PATIENT DOSE REDUCTION BY BISMUTH SHIELDING IN CT USING MEASUREMENTS, GEANT4 AND MCNPX SIMULATIONS
}

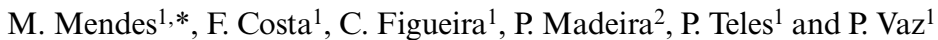 \\ ${ }^{1}$ Centro de Ciências e Tecnologias Nucleares, Instituto Superior Técnico, Universidade de Lisboa, \\ Estrada Nacional 10 (km 139,7), 2695-066 Bobadela LRS, Portugal \\ ${ }^{2}$ Serviço de Radiologia, Hospital de São José, Centro Hospitalar Lisboa Central, EPE, Rua José António \\ Serrano, 1150-199 Lisboa, Portugal \\ *Corresponding author: monicamendes@ctn.ist.utl.pt
}

\begin{abstract}
This work reports on the use of two different Monte Carlo codes (GEANT4 and MCNPX) for assessing the dose reduction using bismuth shields in computer tomography (CT) procedures in order to protect radiosensitive organs such as eye lens, thyroid and breast. Measurements were performed using head and body PMMA phantoms and an ionisation chamber placed in five different positions of the phantom. Simulations were performed to estimate Computed Tomography Dose Index values using GEANT4 and MCNPX. The relative differences between measurements and simulations were $<10 \%$. The dose reduction arising from the use of bismuth shielding ranges from 2 to $45 \%$, depending on the position of the bismuth shield. The percentage of dose reduction was more significant for the area covered by the bismuth shielding (36 \% for eye lens, $39 \%$ for thyroid and $45 \%$ for breast shields).
\end{abstract}

\section{INTRODUCTION}

The use of computed tomography (CT), which started in the early 1970 s, and its dissemination in clinical practice in the following decades very positively impacted diagnostic radiology worldwide ${ }^{(1,2)}$. The introduction of multidetector CT (MDCT) systems provides better image quality with improved spatial resolution but results in higher doses to the patients ${ }^{(1,2)}$. Therefore, it is extremely important to perform the dosimetric assessment of CT examinations and develop methods to reduce patient doses during CT scans.

According to ICRP Publication 103, the tissue weighting factor for breast and thyroid are 0.12 and 0.04 , respectively. The eye lens is also considered an organ at risk to low-dose radiation, especially because of cataract induction ${ }^{(3)}$.

The use of bismuth-impregnated latex shields is a method to protect the organs at risk from direct exposure in CT procedures ${ }^{(4-8)}$. Chang et al. ${ }^{(5)}$ showed the effective performance of bismuth shielding to reduce the unnecessary radiation dose of CT examinations while preserving image quality. Hopper et al. ${ }^{(6)}$ determined a reduction of $50 \%$ in dose to the eye lens during a cranial CT scan.

Monte Carlo simulations can make a very important contribution in assessing absorbed doses in organs, providing information that is not assessed from measurements. In this study, state-of-the-art Monte Carlo computer programs, MCNPX (Monte Carlo N-Particle eXtended) and GEANT4 (GEometry ANd Tracking), are used. The MCNPX code is a well-established method to assess the Computed Tomography Dose Index (CTDI) and absorbed doses in CT scans $5,7,9)$. GEANT4 is an object-oriented software toolkit for the simulation of the transport of particles through matter, and it is used in a variety of application domains, including medical physics and radiation protection. Its functionality and modelling capabilities continue to be studied $^{(10)}$.

The main objectives of this work are (i) to evaluate the effectiveness of bismuth shielding on dose reduction during CT examinations for three different types of shields (eye lens, thyroid and breast) and (ii) to benchmark two MC codes - GEANT4 and MCNPX - in the MC computation of CTDIs for the assessment of radiation dose reduction using bismuth shielding in $\mathrm{CT}$ scans.

\section{MATERIALS AND METHODS}

Dose measurements

The measurements were carried out on a GEC Brightspeed Elite CT scanner using standard head and body PMMA phantoms, with diameter of 16 and $32 \mathrm{~cm}$, respectively, and length of $15 \mathrm{~cm}$ (ProProject $\left.{ }^{\circledR}\right)$. Each phantom has four peripheral holes (designated in sequence at $0^{\circ}, 90^{\circ}, 180^{\circ}$ and $270^{\circ}$ ) and one central hole where a pencil ionisation chamber (IC) RaySafe $^{\mathrm{TM}}$ ) can be placed. Commercial bismuth shields for the eye lens, thyroid and breast (Kiran $(\mathrm{C})$ were utilised in this study $(0.06-\mathrm{mm} \mathrm{Pb}$ equivalence at $120 \mathrm{kVp}$ ). 


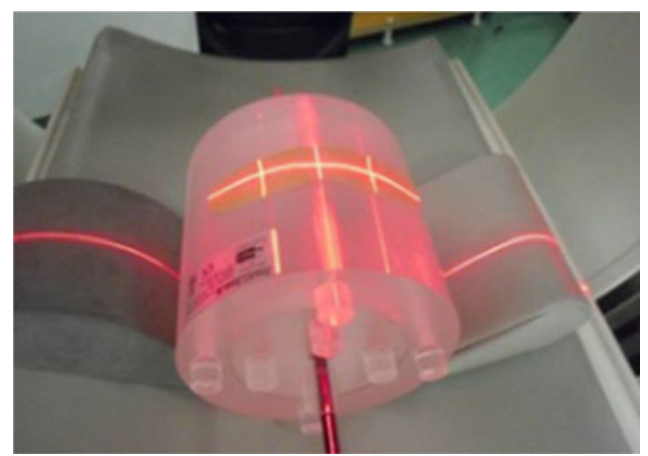

Figure 1. Experimental set-up with the ionisation chamber placed in the central hole of the head phantom with an eye lens bismuth shield.

The measurements were performed five times for each hole with and without bismuth shielding in order to calculate the dose reduction due to the use of shields. The eye and thyroid shields were used for the head phantom and the breast shield for the body phantom. Each phantom was positioned aligning the centre of the phantom with the rotation axis of the gantry (Figure 1). The CT examination was made at $120 \mathrm{kVp}, 200 \mathrm{mAs}$ (head), $300 \mathrm{mAs}$ (body) and $10 \mathrm{~mm}$ of collimation for a complete rotation of $1 \mathrm{~s}$ (one slice).

\section{Computational CT model}

Two MC codes were used for the purpose of this work-GEANT4 and MCNPX. The GEANT4, an object-oriented $\mathrm{C}++$ computer language program, provides comprehensive detector and physics modelling capabilities inserted in a flexible structure ${ }^{(10)}$. MCNPX is a state-of-the-art computer code for particle transport simulation, with the capability of tracking 34 particle types at nearly all energies, and simulation of different geometries using input cards.

The focal spot of the system is located $54 \mathrm{~cm}$ from the centre of the CT gantry. The program SRS-78 $\left(\right.$ IPEM) ${ }^{(11)}$ was used to simulate a typical X-ray energy spectrum of $120 \mathrm{kVp}$. The scanner comprises an X-ray beam filtration that includes both head and body bowtie filters and a collimator. The collimator forces the fan beam to be $55^{\circ}$ in the $x-y$ plane and $10-\mathrm{mm}$ collimation in the $z$ plane. In the absence of detailed information, the shape and dimensions of a typical bowtie were initially implemented in the CT model and its geometry/shape was adjusted by a trial and error iterative method until the relative differences between the measurement and the simulations were $<10 \%$. The CT scanner model simulated in GEANT4 is shown in Figure 2.
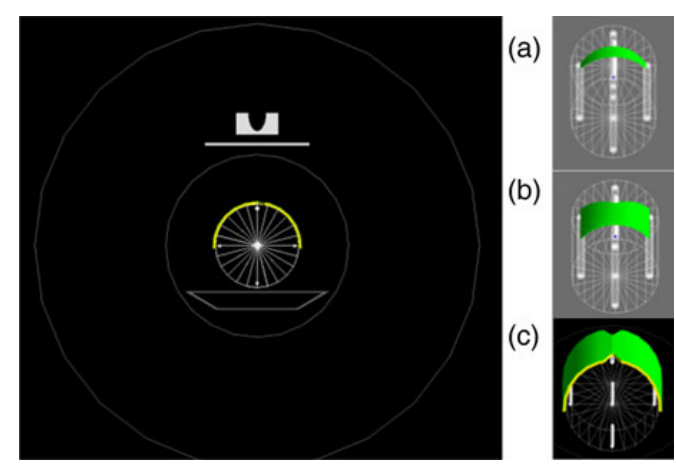

Figure 2. CT scanner set-up developed with GEANT4, and simulated geometries of bismuth shields, (a) eye lens shield, (b) thyroid shield and (c) breast shield.

In the MCNPX code, the tally F6:p results are normalised per source particle. In order to convert the units from MCNPX results (MeV/g/particle) into units of absorbed dose (mGy), a conversion factor is required. The CF used in this study has been described in a previous paper by $\mathrm{Gu}$ et $\mathrm{al}^{(12)}$ as a function of beam energy $\mathrm{E}$ and collimation NT. The CF is defined

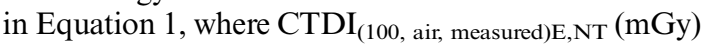
is taken in air by the IC at the scanner isocentre, and $\mathrm{CTDI}_{(100 \text {, air, simulated per particle)E,NT }}\left(\mathrm{Mev} \mathrm{g}^{-1}\right.$ per particle) is obtained by simulating the IC placed on the phantom under the same conditions.

$$
(\mathrm{CF})_{\mathrm{E}, \mathrm{NT}}=\frac{\left(\mathrm{CTDI}_{100, \text { air, measured }}\right)_{\mathrm{E}, \mathrm{NT}}}{\left(\mathrm{CTDI}_{100, \text { air, simulated per particle }}\right)_{\mathrm{E}, \mathrm{NT}}} .
$$

Then, the absolute value for the simulated dose (mGy) is obtained multiplying the simulated dose in $\mathrm{MeV} \mathrm{g}^{-1}$ per particle by the $\mathrm{CF}$ value, as described in the following equation:

$$
D_{\text {absolute }}=D_{\text {simulated }} \times \mathrm{CF} \text {. }
$$

GEANT4 can calculate directly the mean absorbed dose per particle. Nevertheless, in order to know the total absorbed dose in the sensitive volume of the ionisation chamber, the results have to be normalised by the same method described earlier. The geometry and material composition of the bismuth shields were also implemented in GEANT4 and MCNPX.

\section{RESULTS AND DISCUSSION}

\section{Validation of the CT scanner model}

Two sets of measurements were performed in order to validate the $\mathrm{CT}$ scanner model implemented in 
GEANT4 and MCPNX, including the ionisation chamber, the CTDI phantoms and free-in-air measurements. In the first set, a single axial free-in-air measurement using an IC was made, in order to obtain the conversion factor (CF). The second set of measurements used to validate the CT model comprises the CTDI phantom and the IC placed in the centre and in the four peripheral insert positions of the phantom. The measurements were performed for both the head and body phantoms, as well as for the two bowtie filters available (head and body).

The $\mathrm{CTDI}_{100}$ values for measurements and for Monte Carlo simulation obtained with MCNPX and GEANT4 are displayed in Table 1 (for $200 \mathrm{mAs}$ ) and Table 2 (for $300 \mathrm{mAs}$ ) for the head and body phantom without bismuth, respectively. The results of Table 1 show good agreement between simulations and measurements, with a maximum percentage difference of $8 \%$ between GEANT 4 and measurements. The measured $\mathrm{CTDI}_{100}$ for the $0^{\circ}$ position (the position representing superficial organs) of the head phantom was $36.4 \mathrm{mGy}$ for measurements, $34.6 \mathrm{mGy}$ for MCNPX and $34.4 \mathrm{mGy}$ for GEANT4.

Regarding the measurements with the body phantom (Table 2), the value obtained for the $0^{\circ}$ position was 32.9 mGy. With MCNPX and GEANT4, the values for position $0^{\circ}$ were 32.6 and $33.0 \mathrm{mGy}$, respectively. The two MC codes show a maximum percentage difference of $6 \%$

For the measurements without bismuth shielding, the highest dose value was obtained for the position $0^{\circ}$ of the head phantom. The lowest value corresponds to the $180^{\circ}$ position, because the radiation attenuation induced by $\mathrm{CT}$ table. For the simulation studies, this was not verified, and the highest dose value in the centre was slightly $>0^{\circ}$ (Figure 3 ).

\section{Dose measurements with bismuth shielding}

Tables 3 and 4 show the measured and simulated CTDI $_{100}$ values in the head phantom with eye lens and thyroid bismuth shielding, respectively. The relative differences between measurements and simulations are $<10 \%$. The main complication in setting up an MC simulation arises from the need to validate and define the geometry and the experimental set-up with high accuracy. Consequently, the relative differences obtained are mainly due to uncertainties of $X$-ray spectra $(\approx 15 \%)^{(11)}$, filtration, properties of materials and geometry.

Using the eye lens bismuth shielding, the obtained CTDI values for the $0^{\circ}$ position are $23.2 \mathrm{mGy}$ (measurements), $21.9 \mathrm{mGy}$ (MCNPX) and $21.9 \mathrm{mGy}$ (GEANT4). Using the thyroid bismuth shield, the obtained values are $22.0 \mathrm{mGy}$ (measurements), 21.4 mGy (MCNPX) and $21.1 \mathrm{mGy}$ (GEANT4). The last column of the tables indicates a relative difference

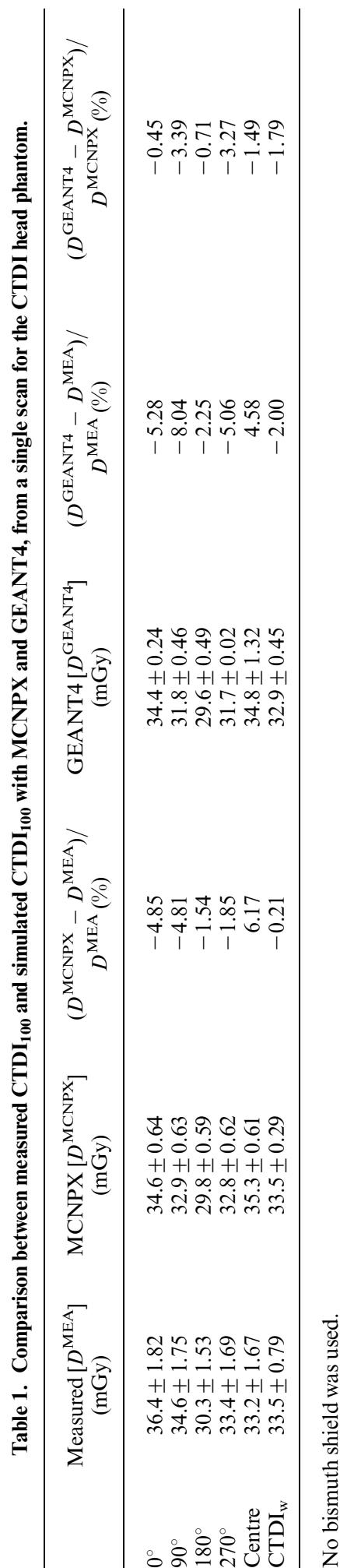




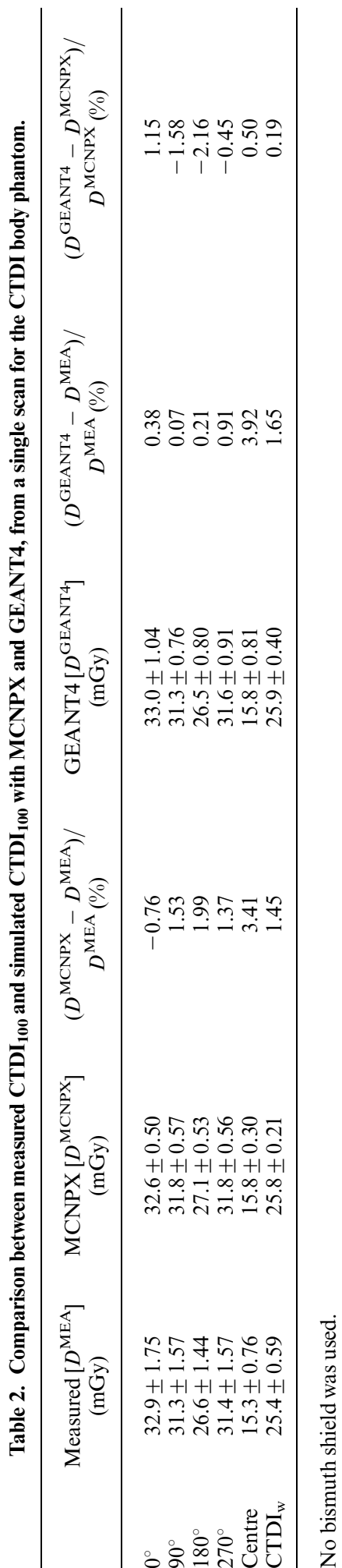

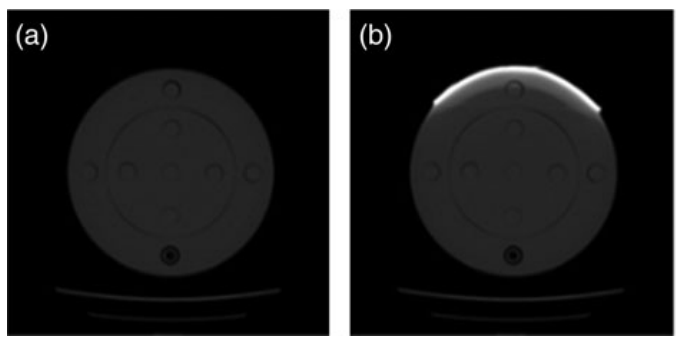

Figure 3. CT scan images of the PMMA head phantom for $120 \mathrm{kVp}$ and $200 \mathrm{mAs}$, (a) without bismuth shield and (b) with an eye lens bismuth shield.

between the two MC programs up to $5 \%$, except for the central hole with the eye lens bismuth shield (relative difference of $\approx 7 \%$ ).

Table 5 presents the CTDI 100 values for measurements and simulations with GEANT4 and MCNPX, in the body phantom with breast shielding. The $\mathrm{CTDI}_{100}$ values for the $0^{\circ}$ position with the breast shield were 17.9, 16.7 and $17.3 \mathrm{mGy}$ for measurements, MCNPX and GEANT4 simulations, respectively.

Figures 4-6 show the percentage of dose reduction using the three different types of bismuth shields. In all cases, the major dose reduction is for the ionisation chamber inserted in the $0^{\circ}$ position, which was expected since this corresponds to the position adjacent to the bismuth shielding. For measurements, the dose reduction at the $0^{\circ}$ position was 36,39 and $45 \%$ for eye lens, thyroid and breast bismuth shields, respectively. The dose reduction at the centre was approximately half of those at $0^{\circ}$, and one-third at the lateral positions $\left(90^{\circ}\right.$ and $\left.270^{\circ}\right)$. These results indicate the effect of the area covered by the shields on the absorbed dose.

In general, there is a good agreement between the measurements and the Monte Carlo simulations for the three cases. Previous studies have demonstrated similar results and conclusions supporting the efficacy of bismuth shields on dose reductions. In fact, McLaughlin et al. ${ }^{(13)}$ recommend the use of thyroid shields in routine practice. Catuzzo et al. ${ }^{(8)}$ demonstrate that the use of a bismuth shield reduces significantly both organ and effective radiation dose with a consequent reduction in the risk for the patient. A good agreement, $<5 \%$, between the GEANT4 and MCNPX Monte Carlo simulation results, is also observed.

Additional work is necessary to corroborate these results and give more detailed information about the dose reduction in the organs of interest. Some authors have demonstrated that other techniques, such as automatic tube modulation and automatic exposure control, show good results in dose reduction without degradation of image quality and could offer a better option than bismuth ${ }^{(14,15)}$. 
Table 3. Comparison between measured $\mathrm{CTDI}_{100}$ and simulated $\mathrm{CTDI}_{100}$ with $\mathrm{MCNPX}$ and GEANT4, from a single scan for the CTDI head phantom with eye lens bismuth shielding.

\begin{tabular}{|c|c|c|c|c|c|c|}
\hline & $\begin{array}{c}\text { Measured }\left[D^{\mathrm{MEA}}\right] \\
(\mathrm{mGy})\end{array}$ & $\begin{array}{c}\operatorname{MCNPX}\left[D^{\mathrm{MCNPX}}\right] \\
(\mathrm{mGy})\end{array}$ & $\begin{array}{c}\left(D^{\mathrm{MCNPX}}-D^{\mathrm{MEA}}\right) / \\
D^{\mathrm{MEA}}(\%)\end{array}$ & $\begin{array}{c}\text { GEANT4 }\left[D^{\text {GEANT4 }}\right] \\
(\mathrm{mGy})\end{array}$ & $\begin{array}{c}\left(D^{\mathrm{GEANT} 4}-D^{\mathrm{MEA}}\right) / \\
D^{\mathrm{MEA}}(\%)\end{array}$ & $\begin{array}{c}\left(D^{\text {GEANT4 }}-D^{\text {MCNPX }}\right) / \\
D^{\operatorname{MCNPX}}(\%)\end{array}$ \\
\hline $0^{\circ}$ & $23.2 \pm 1.17$ & $21.9 \pm 0.48$ & -5.52 & $21.9 \pm 1.21$ & -5.84 & -0.12 \\
\hline $90^{\circ}$ & $30.1 \pm 1.52$ & $30.9 \pm 0.55$ & 2.44 & $30.1 \pm 1.12$ & -0.31 & -2.69 \\
\hline $180^{\circ}$ & $28.8 \pm 1.45$ & $29.1 \pm 0.55$ & 0.94 & $27.6 \pm 0.24$ & -4.07 & -4.96 \\
\hline $270^{\circ}$ & $30.5 \pm 1.54$ & $30.9 \pm 0.56$ & 1.23 & $29.4 \pm 0.68$ & -3.71 & -4.88 \\
\hline Centre & $27.9 \pm 1.42$ & $30.4 \pm 0.37$ & 9.02 & $28.1 \pm 1.04$ & 0.77 & -7.57 \\
\hline $\mathrm{CTDI}_{\mathrm{w}}$ & $28.1 \pm 0.67$ & $28.9 \pm 0.22$ & 3.06 & $27.5 \pm 0.46$ & -1.92 & -4.84 \\
\hline
\end{tabular}

Table 4. Comparison between measured CTDI $_{100}$ and simulated CTDI $_{100}$ with MCNPX and GEANT4, from a single scan for the CTDI head phantom with thyroid bismuth shielding.

\begin{tabular}{|c|c|c|c|c|c|c|}
\hline & $\begin{array}{l}\text { Measured }\left[D^{\mathrm{MEA}}\right] \\
(\mathrm{mGy})\end{array}$ & $\begin{array}{c}\operatorname{MCNPX}\left[D^{\mathrm{MCNPX}}\right] \\
(\mathrm{mGy})\end{array}$ & $\begin{array}{c}\left(D^{\mathrm{MCNPX}}-D^{\mathrm{MEA}}\right) / \\
D^{\mathrm{MEA}}(\%)\end{array}$ & $\begin{array}{c}\text { GEANT4 }\left[D^{\text {GEANT4 }}\right] \\
(\mathrm{mGy})\end{array}$ & $\begin{array}{c}\left(D^{\mathrm{GEANT} 4}-D^{\mathrm{MEA}}\right) / \\
D^{\mathrm{MEA}}(\%)\end{array}$ & $\begin{array}{c}\left(D^{\mathrm{GEANT} 4}-D^{\mathrm{MCNPX}}\right) / \\
D^{\mathrm{MCNPX}}(\%)\end{array}$ \\
\hline $0^{\circ}$ & $22.0 \pm 1.11$ & $21.4 \pm 0.46$ & -2.67 & $21.1 \pm 0.91$ & -4.09 & -1.46 \\
\hline $90^{\circ}$ & $32.4 \pm 1.63$ & $30.7 \pm 0.55$ & -5.20 & $30.4 \pm 0.14$ & -6.20 & -1.06 \\
\hline $180^{\circ}$ & $29.6 \pm 1.49$ & $29.0 \pm 0.53$ & -2.12 & $27.7 \pm 0.12$ & -6.55 & -4.53 \\
\hline $270^{\circ}$ & $32.1 \pm 1.61$ & $30.7 \pm 0.55$ & -4.34 & $29.4 \pm 0.37$ & -8.57 & -4.42 \\
\hline Centre & $27.2 \pm 1.36$ & $29.9 \pm 0.37$ & 10.15 & $28.8 \pm 0.47$ & 5.98 & -3.79 \\
\hline $\mathrm{CTDI}_{\mathrm{w}}$ & $28.4 \pm 0.67$ & $28.6 \pm 0.21$ & 0.70 & $27.7 \pm 0.23$ & -2.57 & -3.25 \\
\hline
\end{tabular}



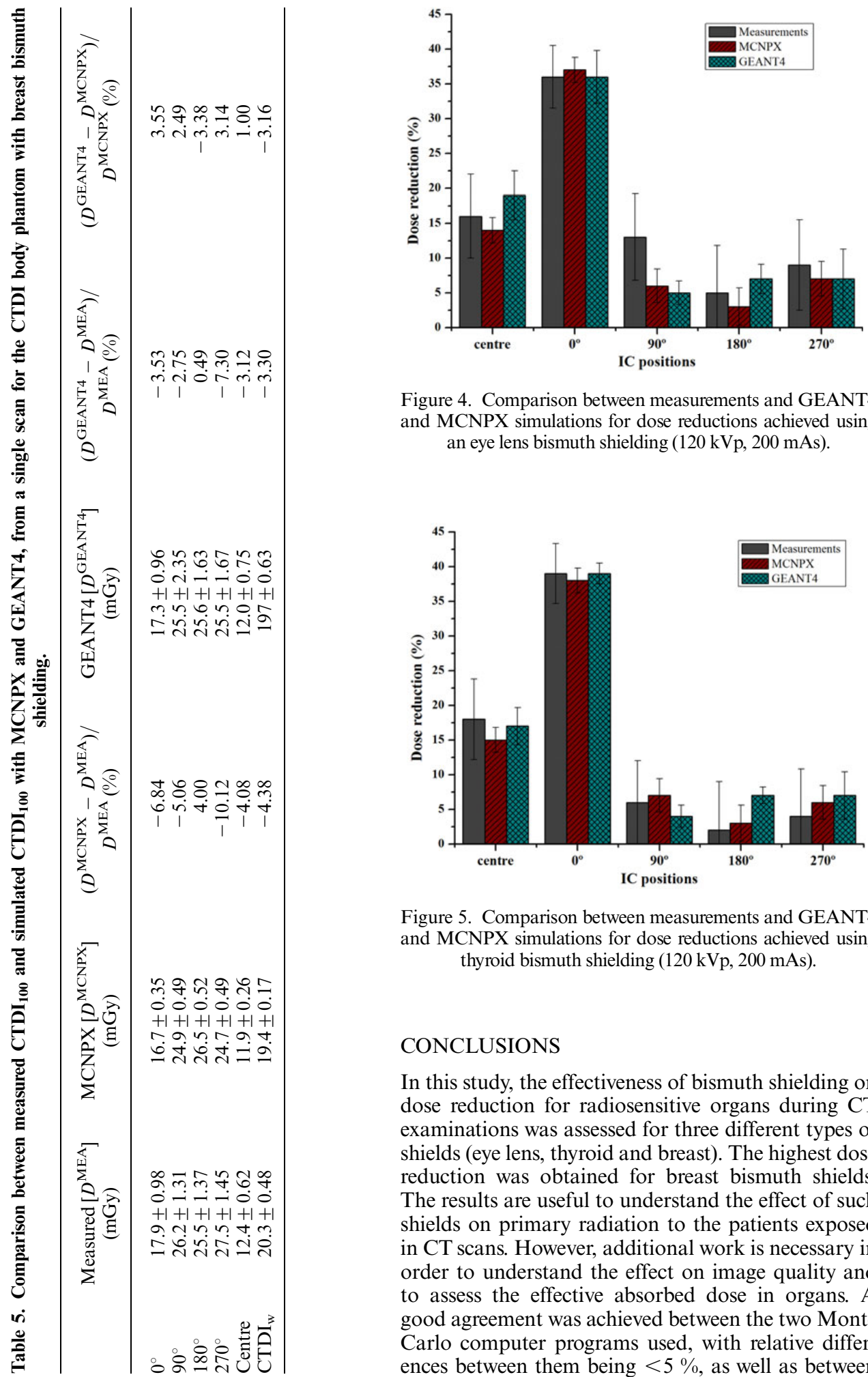

Figure 4. Comparison between measurements and GEANT4 and MCNPX simulations for dose reductions achieved using an eye lens bismuth shielding ( $120 \mathrm{kVp}, 200 \mathrm{mAs})$.

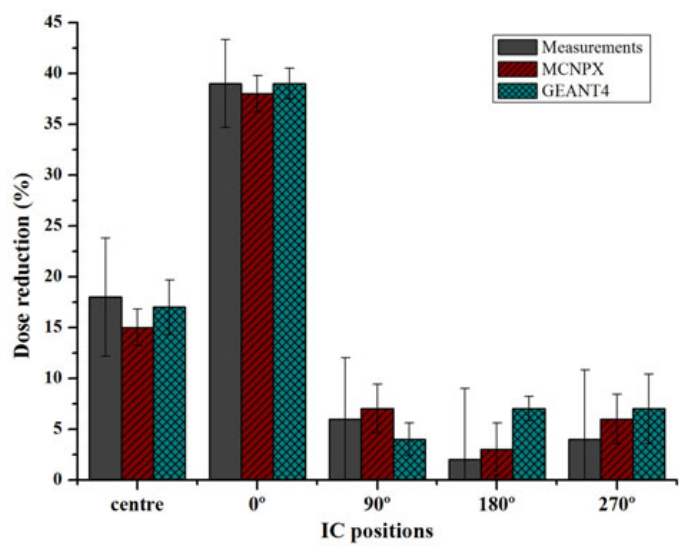

Figure 5. Comparison between measurements and GEANT4 and MCNPX simulations for dose reductions achieved using thyroid bismuth shielding ( $120 \mathrm{kVp}, 200 \mathrm{mAs})$.

\section{CONCLUSIONS}

In this study, the effectiveness of bismuth shielding on dose reduction for radiosensitive organs during CT examinations was assessed for three different types of shields (eye lens, thyroid and breast). The highest dose reduction was obtained for breast bismuth shields. The results are useful to understand the effect of such shields on primary radiation to the patients exposed in CT scans. However, additional work is necessary in order to understand the effect on image quality and to assess the effective absorbed dose in organs. A good agreement was achieved between the two Monte Carlo computer programs used, with relative differences between them being $<5 \%$, as well as between 


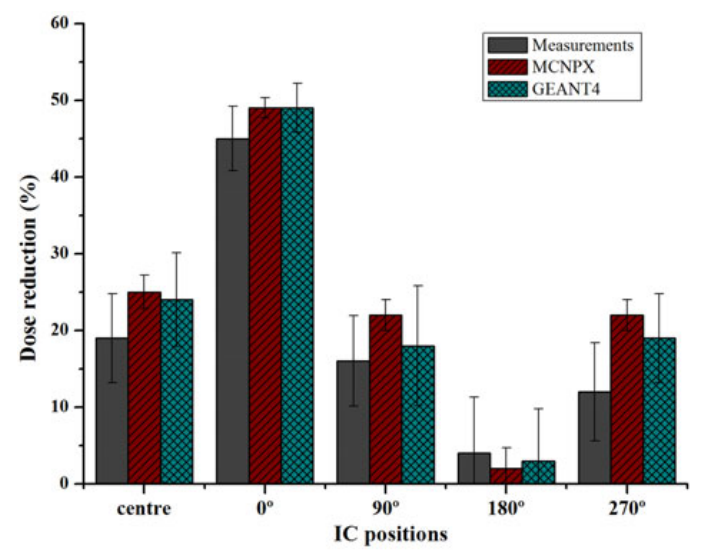

Figure 6. Comparison between measurements and GEANT4 and MCNPX simulations for dose reductions achieved using breast bismuth shielding (120 kVp, $300 \mathrm{mAs})$.

the measured and the simulated doses. Therefore, the validated computational models can be used, in the future, to perform the dosimetric assessment of more complex scenarios including the implementation of human voxel phantoms, tube current modulation and other dose reduction techniques.

\section{ACKNOWLEDGEMENTS}

The authors thank Centro Hospitalar Lisboa Central for kindly making available the CT scan equipment, and Ana Fernandes and Joana Simões for all their support during this work.

\section{REFERENCES}

1. McCollough, C. H. and Zink, F. E. Performance evaluation of a multi-slice CT system. Med. Phys. 26, 2223-2230 (1999).

2. Kohl, G. The evolution and state-of-the-art principles of multislice computed tomography. Proc. Am. Thoracic Soc., 2, 470-476, 499-500 (2005).

3. ICRP. The 2007 Recommendations of the International Commission on Radiological Protection. ICRP Publication 103. Ann. ICRP 37(2-4) (2007).

4. Mukundan, S. Jr., Wang, P. I., Frush, D. P., Yoshizumi, T., Marcus, J., Kloeblen, E. and Moore, M. MOSFET dosimetry for radiation dose assessment of bismuth shielding of the eye in children. AJR. 188(6), 1648-1650 (2007).

5. Chang, K. H., Lee, W., Choo, D. M., Lee, C. S. and Kim, Y. Dose reduction in CT using bismuth shielding: measurements and Monte Carlo simulations. Radiat. Prot. Dosim. 138(4), 382-388 (2010).

6. Hopper, K. D., Neuman, J. D., King, S. H. and Kunselman, A. R. Radioprotection to the eye during CT scanning. AJNR. 22(6), 1194-1198 (2001).

7. DeMarco, J. J., Cagnon, C. H., Cody, D. D., Stevens, D. M., McCollough, C. H., Zankl, M., Angel, E. and McNitt-Gray, M. F. Estimating radiation doses from multidetector CT using Monte Carlo simulations: effects of different size voxelized patient models on magnitudes of organ and effective dose. Phys. Med. Biol. 52(9), 2583-2597 (2007).

8. Catuzzo, P., Aimonetto, S., Fanelli, G., Marchisio, P., Meloni, T., Mistretta, L., Pasquino, M., Richetta, E. and Tofani, S. Dose reduction in multislice CT by means of bismuth shields: results of in vivo measurements and computed evaluation. Radiol. Med. 115, 152-169 (2010).

9. Figueira, C., Becker, F., Blunck, C., DiMaria, S., Baptista, M., Esteves, B., Paulo, G., Santos, J., Teles, P. and Vaz, P. Medical staff extremity dosimetry in CT fluoroscopy: an anthropomorphic hand voxel phantom study. Phys. Med. Biol. 58(16), 5433-5448 (2013).

10. Agostinelli, S. et al. Geant4-a simulation toolkit. Nucl. Instrum. Methods 506(3), 250-303 (2003).

11. Cranley, K., Gilmore, B. J., Fogerty, G. W. and Desponds, L. Catalogue of Diagnostic X-ray Spectra and Other Data. Institute of Physics and Engineering in Medicine. IPEM SRS-78 IPEM (1997).

12. Gu, J., Bednarz, B., Caracappa, P. F. and Xu, X. G. The development, validation and application of a multidetector CT (MDCT) scanner model for assessing organ doses to the pregnant patient and the fetus using Monte Carlo simulations. Phys. Med. Biol. 54(9), 2699-2717 (2009).

13. McLaughlin, D. J. and Mooney, R. B. Dose reduction to radiosensitive tissues in CT. Do commercially available shields meet the users' needs? Clin. Radiol. 59, 446-450 (2004).

14. Vollmar, S. V. and Kalender, W. A. Reduction of dose to the female breast in thoracic CT: a comparison of standard-protocol, bismuth-shielded, partial and tube-currentmodulated CT examinations. Eur. Radiol. 18, 1674-1682 (2008).

15. Wang, J., Duan, X., Christner, J. A., Leng, S., Grant, K. L. and McCollough, C. H. Bismuth shielding, organ-based tube current modulation, and global reduction of tube current for dose reduction to the eye at head ct. Radiol. 262(1), 191-198 (2012). 